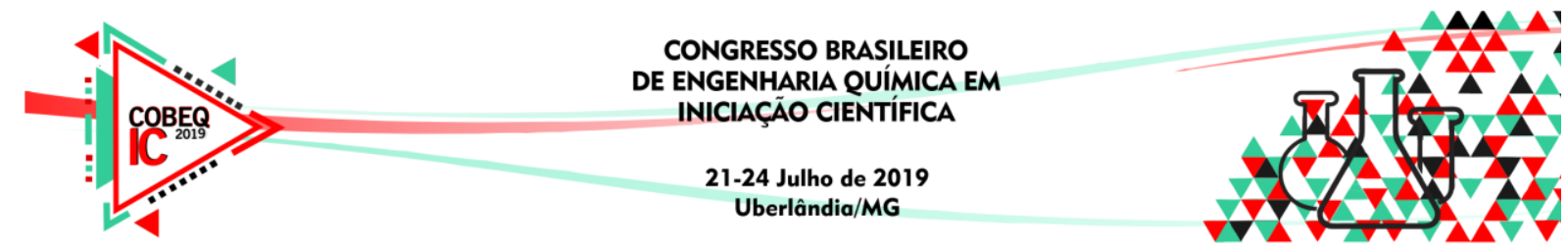

\title{
MEDIÇÃO DE VAZÃO ATRAVÉS DE UM TUBO DE VENTURI - ESTUDO EXPERIMENTAL E NUMÉRICO COMO FERRAMENTA PARA A APRENDIZAGEM DE ENGENHARIA QUÍMICA
}

\author{
F. O. COSTA, C. G. S. FERREIRA, G. K. T. ANDRADE, J. R. SANTOS e R. A. B. LIMA- \\ CORRÊA
}

Universidade Federal de Lavras, Departamento de Engenharia

E-mail para contato: felipe.costa@estudante.ufla.br

\begin{abstract}
RESUMO - O tubo de Venturi é um medidor de vazão de gases e líquidos por obstrução. Apresenta como vantagens uma baixa perda de carga e uma grande precisão comparada a outros medidores, como a placa de orifício e os medidores bocal. Como ferramenta para a consolidação de conceitos fundamentais de Engenharia Química, um aparato experimental para medição de vazão utilizando um tubo Venturi foi desenvolvido ao longo das aulas da disciplina Projetos em Engenharia Química I, da Universidade Federal de Lavras. O sistema de tubulações interligadas com o tubo Venturi foi construído com materiais de fácil acesso e baixo custo. A medição da vazão foi feita utilizando um cronômetro e um recipiente com capacidade de 15 L. Já a variação da pressão foi obtida a partir da variação de altura de água em manômetros confeccionados com mangueiras de silicone. Os resultados experimentais de vazão e variação de pressão foram comparados com valores teóricos e com os obtidos pela aplicação de um método numérico computacional (CFD). O cálculo do coeficiente de descarga resultou em um valor de $0,68 \pm 0,028$, valor intermediário entre os encontrados na literatura para medidores de vazão do tipo placa de orifício e bocal. O modelo numérico utilizado foi capaz de prever de forma satisfatória a perda de carga no sistema.
\end{abstract}

\section{INTRODUÇÃO}

Os medidores de vazão por obstrução, que são amplamente utilizados para medir vazões de gases e líquidos em diversos processos industriais, se baseiam no princípio da aceleração de uma corrente fluida. Dentre os diferentes tipos de medidores de vazão de restrição, o tubo de Venturi combina dentro de uma unidade simples uma 'garganta' estreitada curta entre duas seções cônicas. A ideia é provocar uma variação na velocidade e, como resultado, uma variação na pressão, que possa ser medida com o uso de medidores apropriados (manômetros, por exemplo). Assim, a vazão pode ser inferida, relacionando o diferencial de pressão pela aplicação das equações da continuidade e Bernoulli. Os medidores Venturi se destacam pelas baixas perdas de carga impostas ao sistema e pela elevada precisão. Em geral, estes medidores são fundidos e usinados com tolerâncias pequenas em relação ao projeto-padrão e, como resultado, são volumosos e caros (Fox et al., 2018). 


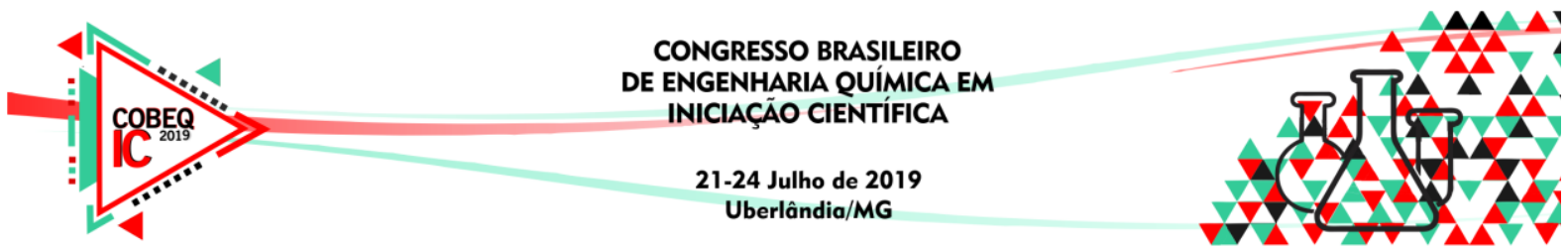

Com o intuito de consolidar conceitos fundamentais de Engenharia Química, uma linha experimental simples para medição de vazão utilizando um 'tubo Venturi' foi desenvolvida ao longo das aulas da disciplina Projetos em Engenharia Química I, do curso de graduação em Engenharia Química da Universidade Federal de Lavras (UFLA). De forma a complementar os resultados experimentais obtidos, foi desenvolvido um modelo matemático, que foi simulado através do método dos elementos finitos, usando o CFD (Computational Fluid Dynamics). A aplicação do método numérico pode ser dividida em três etapas principais, as quais, na nomenclatura do método de elementos finitos, são: pré-processamento, processamento e pós-processamento. $\mathrm{Na}$ etapa de pré-processamento, define-se o domínio geométrico e, posteriormente, é feita a discretização do domínio, formando assim a malha. Nesta etapa podem ser definidas condições de contorno e iniciais necessárias para a solução do problema. Na fase de processamento, aplica-se um "solver" baseado no modelo numérico para a obtenção das grandezas de interesse. Na etapa de pós-processamento, os resultados são analisados para se verificar a qualidade do modelo numérico, através de um comparativo com os resultados experimentais (Lima, 2005).

Assim, com o intuito de melhor compreender alguns conceitos envolvidos na medição de vazão em escoamentos internos, o presente trabalho teve como objetivo a construção e operação de um aparato experimental para medidas de vazão utilizando um 'tubo Venturi'. O aparato foi construído utilizando materiais de fácil acesso e baixo custo. A vazão experimental foi medida pelo método direto e comparada com a vazão teórica. Além disso, a pressão antes e no estrangulamento foi aferida e resultados numéricos obtidos e comparados aos valores experimentais.

\section{MATERIAIS E MÉTODOS}

\section{1 - Materiais}

O aparato experimental construído para as medidas de vazão utilizando um tubo Venturi é mostrado na fotografia da Figura 1. Para a montagem desse aparato foram usados materiais de baixo custo, facilmente adquiridos no comércio local de Lavras, MG. Esses materiais foram: cesto de lixo de $30 \mathrm{~L}$ usado como reservatório de água (a); registro esfera 25 $\mathrm{mm}$ (b); tubo de $25 \mathrm{~mm}$ (c); tubo de $20 \mathrm{~mm}$ (d); bucha redutora $25 \times 20 \mathrm{~mm}$ (e), e mangueiras de silicone (f), além de outros materiais como massa adesiva, fita veda rosca, réguas e abraçadeiras usadas para a construção do manômetro. O custo total foi de $\mathrm{R} \$ 71,59$.

\section{2 - Metodologia Experimental}

Para a montagem do aparato mostrado na Figura 1, um tubo $(D=25 \mathrm{~mm})$ com $0,10 \mathrm{~m}$ de comprimento foi afixado em um orifício criado no balde, e a ele fixou-se o registro esfera. Ao registro esfera foi conectada uma seção de $0,45 \mathrm{~m}$ do tubo de $25 \mathrm{~mm}$, seguido pela bucha redutora, seção de $0,30 \mathrm{~m}$ do tubo menor $(\mathrm{D}=20 \mathrm{~mm})$, segunda bucha redutora e, por fim, a seção de $0,15 \mathrm{~m}$ do tubo maior, formando a estrutura principal do tubo de Venturi.

As medidas experimentais de vazão (Q) foram realizadas pelo método direto (seis réplicas), medindo-se o volume de água escoada em um recipiente em um dado intervalo de tempo. A vazão volumétrica teórica $\left(Q_{\text {teórica}}\right)$ foi calculada pelo uso da Equação (1), obtida a partir da aplicação das equações da continuidade e de Bernoulli, supondo: regime permanente, 


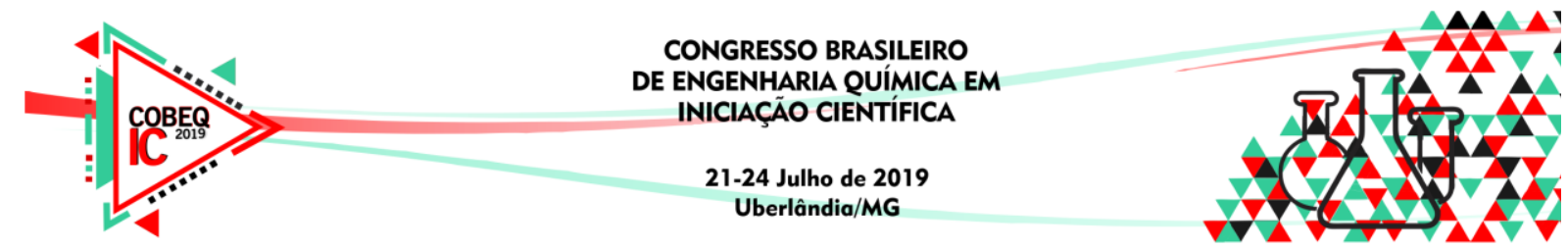

fluido incompressível, escoamento ao longo de uma linha de corrente, escoamento sem atrito, velocidades uniformes nas seções e diferença de cota desprezível. Nesta equação, $\Delta \mathrm{P}$ é a queda de pressão causada pela passagem do fluido através do medidor Venturi, $\mathrm{D}_{1}$ e $\mathrm{D}_{2}$ são diâmetros característicos de um escoamento interno, conforme representado na Figura 2 para um escoamento através de um bocal genérico. A queda de pressão em (1) é obtida a partir da diferença de altura do fluido nas mangueiras de silicone $(\Delta \mathrm{h})$, utilizando a Equação (2).

Figura 1- Aparato experimental para medição de vazão.

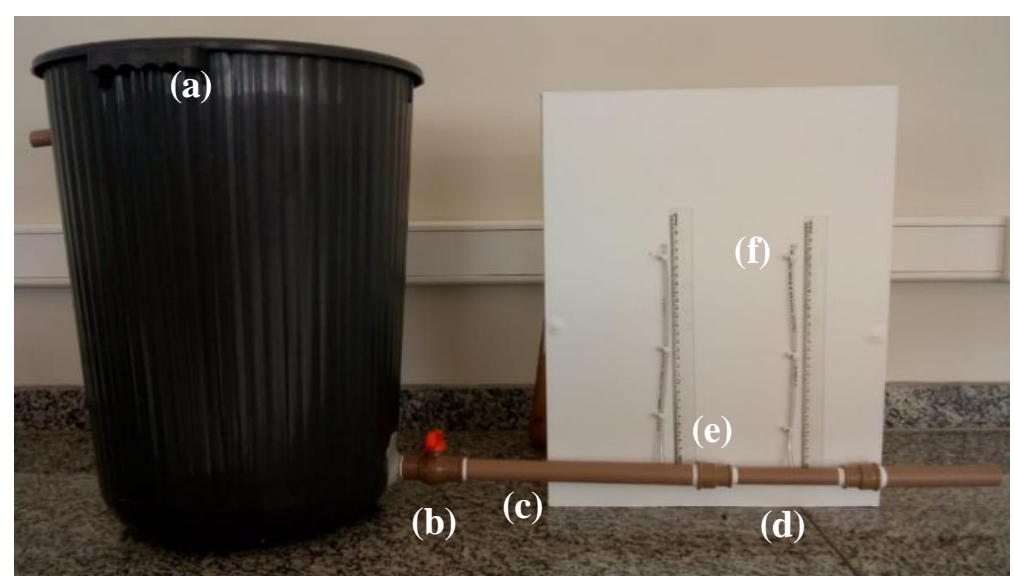

$Q_{\text {teórica }}=A_{t}\left\{\frac{2 \Delta P}{\rho\left[1-\left(\frac{D_{t}}{D_{1}}\right)^{4}\right]}\right\}^{\frac{1}{2}}$

$\Delta P=\rho g \Delta h$

Figura 2 - Escoamento interno através de um bocal genérico (Fonte: Fox et al., 2018).

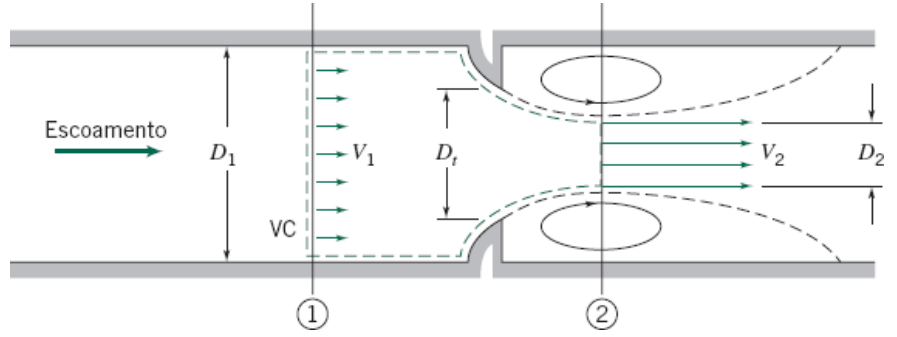

A vazão em massa teórica ( $\dot{m}_{\text {teórica }}$ ) é dada pela Equação (3), também representada em função de $\Delta \mathrm{P}, \mathrm{D}_{1}$ e $\mathrm{D}_{2}$. O coeficiente de descarga $\left(\mathrm{C}_{\mathrm{d}}\right)$ é inserido devido a inviabilidade de se obter o diâmetro do escoamento de fluido na região $2\left(\mathrm{D}_{2}\right)$. Dessa forma, faz-se necessário inserir esse fator de correção para o cálculo da vazão real ( $\dot{m}_{\text {real }}$ ), com o diâmetro da restrição $\left(D_{t}\right)$. A Equação (4) é utilizada para o cálculo de $C_{d}$.

$$
\begin{aligned}
& \dot{m}_{\text {teórica }}=A_{t}\left\{\frac{2 \rho \Delta P}{\left[1-\left(\frac{D_{t}}{D_{1}}\right)^{4}\right]}\right\}^{\frac{1}{2}} \\
& C_{d}=\frac{\dot{m}_{\text {real }}}{\dot{m}_{\text {teorico }}}=\frac{Q_{\text {real }}}{Q_{\text {teorico }}}
\end{aligned}
$$




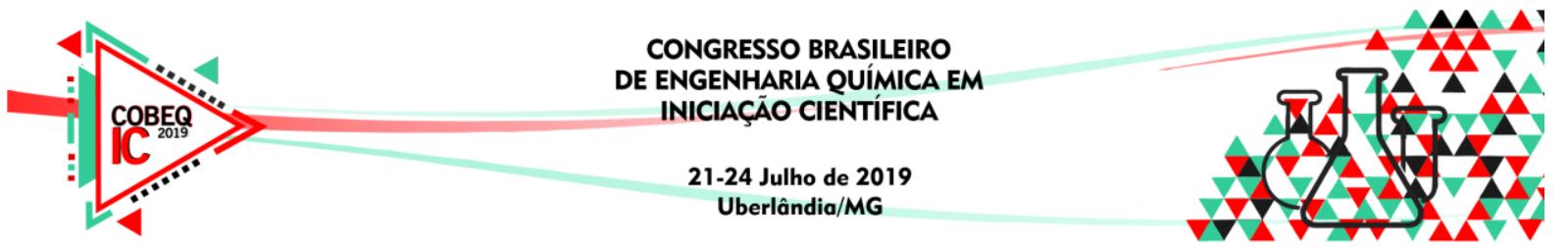

\section{3 - Solução Numérica}

O domínio da solução numérica é dividido em pequenos volumes de controle para que posteriormente as equações de transporte possam ser aplicadas (VIEIRA, 2006). A manufatura da geometria e das malhas computacionais advém do software comercial GAMBIT $^{\circledR}$. Durante as simulações, a estrutura base das malhas é utilizada pelo software FLUENT $^{\circledR} 19.0$ pertencente ao pacote ANSYS. A versão acadêmica foi utilizada. As condições de contorno utilizadas no software FLUENT $^{\circledR}$ estão contidas na Tabela 1.

Tabela 1 - Condições de Contorno no software FLUENT ${ }^{\circledR}$.

Simulação 3D

Regime Transiente

Saída: Pressão atmosférica (100700 Pa)

Vazão de alimentação: $537,31 \mathrm{~cm}^{3} / \mathrm{s}$

Modelo de Turbulência: Large Eddy Simulation (LES)

Método de discretização do momento: QUICK

Método de discretização da Pressão: PRESTO!

Fatores de relaxação (Petri et al., 2009)

Pressão $=0,3$

Densidade $=1$

Body Force $=1$

Momentum $=0,1$

Critério de Convergência $=10^{-4}$

Time StepSize $(\mathrm{s})=10^{-5}$

\section{RESULTADOS E DISCUSSÕES}

Inicialmente, é importante destacar que o aparato construído foi adequado para a medição dos dados experimentais ( $\mathrm{Q}$ e $\Delta \mathrm{h}$ ). $\mathrm{O}$ valor médio obtido para a vazão experimental foi de $(0,537 \pm 0,014) \mathrm{L} / \mathrm{s}$. O baixo valor de desvio padrão sugere uma boa reprodutibilidade do procedimento experimental utilizado.

A perda de carga do fluido $(\Delta \mathrm{P})$ ao atravessar o medidor Venturi foi obtida a partir da diferença de altura de água $(\Delta \mathrm{h})$ em mangueiras de silicone apoiadas verticalmente (ver Figura 1), através do uso da equação (2). Os valores de $\Delta \mathrm{h}$ medidos e as respectivas perdas de carga calculadas estão apresentadas juntamente com o valor médio e desvio padrão na Tabela 2 .

Tabela 2 - Valores medidos da diferença de altura e pressão

\begin{tabular}{|c|c|c|}
\hline Medições & $\Delta \mathrm{h}(\mathrm{cm})$ & $\Delta \mathrm{P}(\mathrm{Pa})$ \\
\hline \hline 1 & 0,185 & 1811,583 \\
\hline 2 & 0,187 & 1831,168 \\
\hline 3 & 0,189 & 1850,753 \\
\hline 4 & 0,188 & 1840,960 \\
\hline 5 & 0,191 & 1870,337 \\
\hline 6 & 0,192 & 1880,130 \\
\hline Valor médio & $0,189 \pm 0,002$ & $1847,49 \pm 23,08$ \\
\hline
\end{tabular}




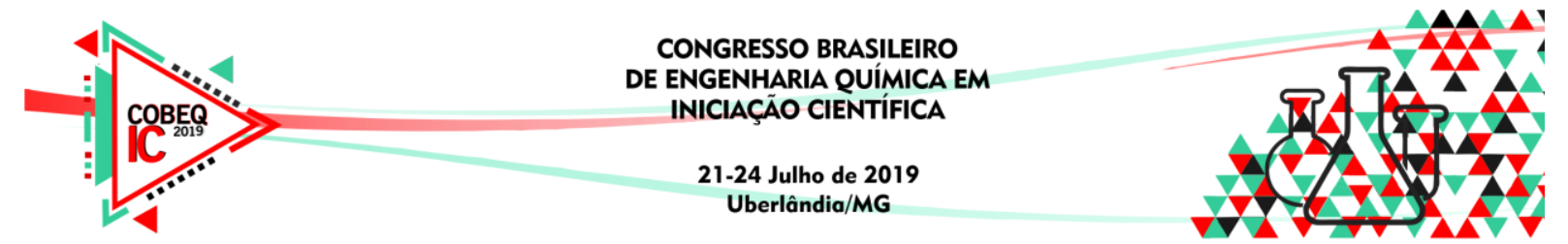

Com os valores da vazão experimental, diferença de altura e diferença de pressão do fluido, é possível obter a vazão teórica, bem como o coeficiente de descarga, pelo uso das equações (3) e (4), respectivamente. Os resultados são apresentados na Tabela 3. Uma análise dos resultados mostra que há uma diferença significativa entre a vazão experimental e a teórica. Essa diferença pode ser atribuída às incertezas nas medições dos dados experimentais, por exemplo, no volume de água escoada e no valor de $\Delta \mathrm{h}$ lido no manômetro. Além de serem análises subjetivas, algumas dificuldades como a oscilação do menisco no manômetro podem ter contribuído para essa divergência entre os valores de vazão. $\mathrm{O}$ valor obtido para $\mathrm{C}_{\mathrm{d}}$ foi intermediário aos encontrados para medidores de vazão por placa de orifício $(=0,61)$ e medidores de bocal, cujo $\mathrm{C}_{\mathrm{d}} \approx 0,98$. Esse resultado intermediário pode ser explicado pelas reduções utilizadas, que são muito mais bruscas que as reduções encontradas em tubos de Venturi e bocais medidores tradicionais; contudo, mais suaves que as reduções de placa de orifício.

Tabela 3 - Valores calculados da vazão teórica e do coeficiente de descarga.

\begin{tabular}{|c|c|c|c|c|}
\hline Medições & $\Delta \mathrm{P}(\mathrm{Pa})$ & $\begin{array}{c}\text { Vazão experimental } \\
(\mathrm{L} / \mathrm{s})\end{array}$ & $\begin{array}{c}\text { Vazão teórica } \\
(\mathrm{L} / \mathrm{s})\end{array}$ & $\begin{array}{c}\text { Coeficiente de } \\
\text { descarga }(\mathrm{Cd})\end{array}$ \\
\hline \hline 1 & 1811,58 & 0,538 & 0,779 & 0,691 \\
\hline 2 & 1831,17 & 0,529 & 0,783 & 0,676 \\
\hline 3 & 1850,75 & 0,522 & 0,787 & 0,663 \\
\hline 4 & 1840,96 & 0,539 & 0,785 & 0,686 \\
\hline 5 & 1870,34 & 0,567 & 0,791 & 0,717 \\
\hline 6 & 1880,13 & 0,530 & 0,794 & 0,668 \\
\hline Valor médio & $1847,49 \pm 23,08$ & $0,537 \pm 0,014$ & $0,787 \pm 0,005$ & $0,683 \pm 0,018$ \\
\hline
\end{tabular}

Para a simulação no software FLUENT $^{\circledR}$, foi feita uma geometria e malha utilizando o software GAMBIT ${ }^{\circledR}$. A malha utilizada contém 510000 células. O tempo simulado foi definido como aproximadamente cinco vezes o tempo de residência da água no sistema. Foram definidos também dois planos nos pontos onde a pressão foi aferida no protótipo, para que fosse possível aferir a pressão média nestas seções transversais e, com isso, obter a diferença de pressão simulada pelo software. Com isso, foram simulados em torno de 1,5 segundos de escoamento, e o gráfico da diferença de pressão em função do tempo de simulação decorrido pode ser observado na Figura 3. O valor médio obtido da diferença de pressão na simulação do intervalo de tempo da simulação de 1,2 a $1,5 \mathrm{~s}$ comparado com o valor médio experimental obtido anteriormente pode ser observado na Tabela 4. É possível perceber que os resultados foram próximos, indicando uma boa qualidade do modelo numérico utilizado.

Figura 3 - Gráfico da diferença de pressão simulada em função da experimental.

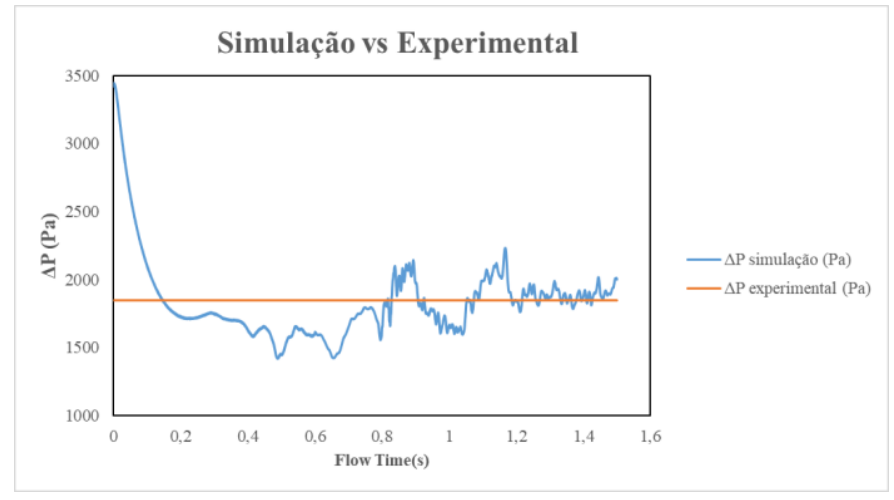




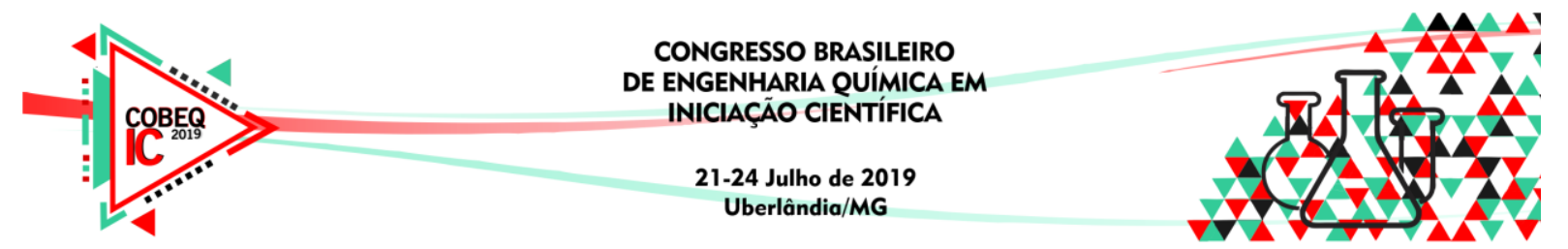

Tabela 4 - Valor médio da diferença de pressão simulada e experimental.

\begin{tabular}{|c|c|c|}
\hline & $\begin{array}{c}\Delta \mathrm{P} \text { simulação } \\
(\mathrm{Pa})\end{array}$ & $\begin{array}{c}\Delta \mathrm{P} \text { experimental } \\
(\mathrm{Pa})\end{array}$ \\
\hline \hline Média & 1886,72 & 1847,50 \\
\hline Desvio padrão & 50,23 & 23,08 \\
\hline
\end{tabular}

\section{CONCLUSÕES}

A construção do aparato experimental para estudo da vazão utilizando um medidor Venturi foi adequada ao estudo proposto, permitindo a obtenção de dados e melhorando a compreensão acerca de conhecimentos teóricos anteriormente adquiridos no curso de Engenharia Química. Valores de vazão experimentais $(0,537 \pm 0,014 \mathrm{~L} / \mathrm{s})$ foram obtidos e comparados a valores teóricos $(0,787 \pm 0,005)$. Valores de $\Delta \mathrm{h}$, também reprodutíveis, foram medidos e utilizados para o cálculo da perda de carga na linha experimental. O estudo experimental permitiu a determinação de valores para $C_{d}$, com uma variação de $43,68 \%$ em relação ao valor encontrado na literatura $\left(\mathrm{C}_{\mathrm{d}}=0,98\right)$.

Os valores experimentais de $\Delta \mathrm{P}$ mostraram-se similares aos obtidos por simulação computacional. Este resultado sugere que, apesar da vazão experimental ter tido uma discrepância maior com o valor teórico, e ainda que os materiais utilizados na construção do aparato experimental influenciem no resultado obtido (como a redução não tão suavizada da restrição, diferente do que ocorre nos tubos de Venturi comerciais), as características do escoamento através do Venturi construído puderam ser adequadamente simuladas.

\section{AGRADECIMENTOS}

Os autores agradecem o apoio do Departamento de Engenharia da Universidade Federal de Lavras e ao professor Irineu Petri Júnior pelo suporte no desenvolvimento do trabalho.

\section{REFERÊNCIAS}

FOX, R. W.; MCDONALD, A. T.; PRITCHARD, P. J.; MITCHELL, J. W. Introdução à mecânica dos fluidos, 9 ed. Rio de Janeiro: LTC, 2018.

LIMA, R. C. Simulação de Grandes Escalas de Escoamentos Incompressíveis com Transferência de Calor e Massa por um Método de Elementos Finitos e Subdomínios. Dissertação | Faculdade de Engenharia de Ilha Solteira - UNESP, São Paulo, 2005.

PETRI, I. J.; DUARTE, C. R.; VIEIRA, L. G. M. Aplicação das técnicas de CFD para predição da distribuição de vazões volumétricas em hidrociclones dispostos em distintas baterias. ENEMP, 2009.

VIEIRA, L. G. M. Otimização dos Processos de Separação em Hidrociclones Filtrantes. Tese (Doutorado) | Universidade Federal de Uberlândia, Uberlândia, 2006. 